\title{
Du salon au boulevard. Un aspect de la mélodie française entre 1870 et 1930
}

\author{
Yves RASSENDREN (Grenoble) ${ }^{1}$
}

\section{Summary}

While the Germanic Lied draws its roots from popular tradition and evolves towards the Kunstlied, French melody, born in refinement, reflects a lighter vein at the turn of the 20th century.

In the post-war context after 1870 , French music seeks an authentically nationalist aesthetic, free of Germanic influence. The nature of melody becomes an important area of exploration and research for composers. Inspired by romantic poetry but also by the literary movements of the late 19th century - Parnassianism and Symbolism - the genre appeals to an intellectual and artistic elite. Melody thus flourishes in concerts and in private Parisian salons.

The historic and social context gives rise to a whole repertoire of political and nationalist songs that contain an element of banter or gouaille. Attracted by the songs of cabaret or boulevard, some composers, seeking to break out of the narrow confines of 'salon' melody propose a new direction for the genre.

Melodies explore the humorous ironic, light-hearted or vulgar domains, occasionally verging on the sensual or bawdy. These works set to music unpretentious texts, occasionally written by the composers themselves.

Among the composers of the 'erudite tradition' who are attracted by this light-hearted and popular vein, the most notable are Chabrier, Satie, Hahn and Poulenc, while several others adopt this trend more intermittently. The nature of the texts, the venues where they are performed and the popularity of the singers contribute to this renaissance of the melody genre.

This article therefore evokes a little-known aspect of the development of melody in France after 1870 .

La mélodie, pièce vocale qui met en musique un poème, s'épanouit de façon éblouissante en France durant la deuxième moitié du $\mathrm{XIX}^{\mathrm{e}}$ siècle ; innombrables sont les poètes dont les vers connaissent une autre dimension grâce à la musique. Les compositeurs exploitent alors les plus grands poètes du XIX ${ }^{\mathrm{e}}$ siècle et du tournant du siècle. Le contexte politique, militaire et social de la fin du XIX ${ }^{\mathrm{e}}$ siècle et du début du $\mathrm{XX}^{\mathrm{e}}$ contribue à bouleverser la vie 
artistique française; dans cet environnement surgissent de nouvelles esthétiques littéraires et musicales, mais de nouvelles affinités entre les deux arts se nouent également.

Ces lignes étudieront tout d'abord les rapports entre textes et musiques dans le répertoire français de cette période - la question rémanente de la proximité ou de l'incompatibilité de ces deux arts, puis analyseront l'évolution des choix littéraires des musiciens dans cette période. Mais en exorde, laissons la parole à Claude Debussy :

Les rapports du vers et de la musique ? Je n’y ai pas pensé. Je m’occupe très peu de musique. Les musiciens et les poètes qui parlent toujours de musique et de poésie me semblent aussi insupportables que les gens de sport qui parlent toujours de sport.

Et d'abord, la vérité on ne peut pas la dire. Vous voulez la savoir ? Eh bien! C'est qu'en effet les musiciens qui ne comprennent rien aux vers ne devraient pas en mettre en musique. Ils ne peuvent que les gâcher... [...] Les vrais beaux vers, il ne faut pas exagérer, il n'y en a pas tant que ça. Qui en fait aujourd'hui ? Mais quand il s'en trouve, il vaut mieux ne pas y toucher. Henri de Régnier, qui fait des vers pleins, classiques, ne peut pas être mis en musique. Et voyez-vous de la musique sur des vers de Racine ou de Corneille ? Seulement, aujourd'hui, les jeunes musiciens ne veulent voir à côté de leur nom que des signatures célèbres...

Et puis, en musique, dites-moi à quoi ça sert, les vers ? A quoi ? On a plus souvent mis de la belle musique sur de mauvaises poésies que de mauvaise musique sur des vrais vers. Les vrais vers ont un rythme propre qui est plutôt gênant pour nous. Tenez, dernièrement, j'ai mis en musique, je ne sais pas pourquoi, trois ballades de Villon... $\mathrm{Si}$, je sais pourquoi : parce que j'en avais envie depuis longtemps. Eh bien, c'est très difficile de suivre bien, de plaquer les rythmes tout en gardant une inspiration. Si on fait de la fabrication, si on se contente d'un travail de juxtaposition, évidemment ce n'est pas difficile, mais alors ce n'est pas la peine. Les vers classiques ont une vie propre, un dynamisme intérieur, pour parler comme les Allemands, qui n'est pas du tout notre affaire.

Avec la prose rythmée, on est plus à son aise, on peut mieux se retourner dans tous les sens. Si le musicien devait faire lui-même sa prose rythmée ? Wagner faisait ainsi ; mais les poèmes de Wagner, c'est comme sa musique, ça n'est pas un exemple à suivre. Ses livrets ne valent pas mieux que d'autres. C'est pour lui qu'ils valaient mieux. Et c'est le principal.

Pour conclure, laissons les grands poètes tranquilles. D'ailleurs, ils aiment mieux ça... En général, ils ont très mauvais caractère. (Debussy 1987, 202)

\section{Remise en cause des rapports musique/poésie}

Debussy souligne dans cette interview la difficulté de mettre en musique un poème versifié, mais de façon plus générale, la difficulté de "garder l'inspiration » musicale face au texte 
poétique. Quant à Henri Duparc, il s'interroge sur la pertinence d'ajouter de la musique aux poèmes :

J'ai fait quelques mélodies dans lesquelles j'ai simplement mis mon âme avec sincérité ; c'est le seul mérite. [...] Pour moi, ma musique inspirée par une poésie n’a de raison d'être que si elle la rend plus touchante pour les âmes qu'émeut l'expression musicale ; mais il y a des poésies parfaites et qui sont tellement... pleines, dirais-je, que la musique - même la plus belle, même celle que je ne peux pas faire - ne peut que les diminuer. $^{2}$

Après avoir resitué le contexte musical du tournant du siècle, nous verrons comment vont coexister plusieurs manières d'envisager le choix des poèmes chez les compositeurs : un goût pour le raffinement et la sophistication hérité de la tradition poétique du XIX siècle d'une part, une sensibilité aux nouvelles inspirations poétiques d'autre part. Nous nous pencherons dans un dernier temps, sur un aspect typiquement français de la mélodie, à mi-chemin entre salon et boulevard.

\section{Contexte historique et musical}

Tandis que le Lied germanique puise ses racines dans l'élément populaire et évolue vers le Kunstlied, la mélodie française, née dans le raffinement se laisse séduire par une veine plus légère au tournant des $\mathrm{XIX}^{\mathrm{e}}$ et $\mathrm{XX}^{\mathrm{e}}$ siècles. Dans le contexte de l'après-guerre de 1870, la musique française se cherche une esthétique authentiquement nationale, affranchie de toute emprise germanique. Le genre de la mélodie devient un champ d'exploration et de recherche essentiel pour les compositeurs. Sollicitant la poésie romantique mais aussi les mouvements littéraires de la fin du XIX ${ }^{\text {e }}$ siècle - Parnasse et Symbolisme, le genre s'adresse à une élite intellectuelle et artistique. La mélodie s'épanouit alors au concert et dans les salons parisiens. Dans cette période où la musique française se cherche une esthétique spécifiquement nationale persiste pourtant une emprise de la musique germanique - le wagnérisme sévit ; beaucoup de compositeurs français semblent déchirés entre leur vénération pour Wagner et le rejet de tout art germanique.

Le contexte nationaliste incite les compositeurs à aborder la mélodie comme un genre spécifiquement français contrebalançant la place du Lied germanique. La Société Nationale de Musique, à la devise hautement éloquente Ars gallica, a été fondée en 1871 par SaintSaëns et Bussine pour promouvoir la musique française et contrecarrer la toute-puissance de la musique germanique dans le contexte de l'après-guerre de 1870. C'est à la SNM que seront créées beaucoup de mélodies des compositeurs français de cette époque : les concerts de musique symphonique, très coûteux, laissent souvent la place à des concerts de musique de chambre ou de mélodie. Un vaste répertoire de mélodies fleurit alors avec Fauré, Duparc, Chausson, Debussy et tant d'autres... 


\section{Les salons bourgeois et aristocratiques}

Au cours de la III ${ }^{e}$ République, Paris voit fleurir un nombre considérable de salons privés ; très souvent tenus par des femmes, aristocrates cultivées ou de la grande bourgeoisie, ces salons jouent un rôle essentiel pour la carrière des artistes et pour la diffusion des œuvres. Parmi les principaux salons parisiens l'on compte ceux de Marguerite de SaintMarceaux, de la Comtesse de Greffulhe et surtout de la Princesse Edmond de Polignac ou de Madeleine Lemaire. Marguerite de Saint-Marceaux et la Princesse Edmond de Polignac, très bonnes musiciennes, instrumentistes et chanteuses, se produisent occasionnellement lors de ces soirées privées. L'élite de la société parisienne de l'époque y côtoie les artistes : écrivains, peintres, compositeurs, interprètes... Ces réunions mondaines permettent de pallier l'insuffisance de structures publiques mais offrent surtout des lieux de rencontres pour un certain milieu social. Si l'intérêt pour les arts anime souvent les hôtes, d'autres motivations justifient parfois l'émergence et l'attrait de ces salons : l'envie de tenir une place dans cette société bourgeoise et aristocratique et de côtoyer le milieu de l'art, et donc par là-même, penser en faire partie, sorte de vanité sociale. Malgré le très bon niveau musical des hôtesses de ces salons, cette société n'est souvent pas exempte d'un fort snobisme culturel. Dans Un amour de Swann, en dépeignant le salon de Madame Verdurin, Proust dénonce avec une ironie mordante les conventions bourgeoises de ces réceptions, l'emprise des modes musicales, le ridicule des attitudes et des normes.

C'est dans ces salons que les artistes, compositeurs, poètes, peintres, cherchent à se faire connaître ; un public très aisé et cultivé, parfois curieux de modernité, s'y presse, jugeant les nouvelles créations de façon péremptoire.

Si quelques compositeurs fréquentent ces salons et y évoluent avec aisance, par habitude sociale, par amitié ou par goût pour ces rencontres musicales - Fauré ou Reynaldo Hahn -, d'autres entrevoient principalement la possibilité de faire connaître leurs œuvres en avant-première, comme galop d'essai précédant la création publique. Ce sera parfois le cas pour Debussy, mais plus fréquemment pour Ravel, Satie, Poulenc et tant d'autres.

Alors qu'une réelle amitié et une complicité artistique lient Fauré à la Princesse de Polignac, Satie au contraire, semble évoluer dans ce monde pour d'autres raisons : toujours confronté aux difficultés financières et animé d'idées politiques très éloignées de celle de cette société aisée, il bénéficie d'une aide matérielle à travers l'accueil que lui offrent dans leurs salons respectifs, Paulette Darty (après 1910), Germaine Bongard ou le Comte Étienne de Beaumont.

Dès 1917, mais surtout après la Première Guerre Mondiale, Étienne de Beaumont accueille en son hôtel, rue Masseran, de très nombreux artistes, acteurs de la modernité : Picasso, Braque, Gris, Cocteau, Satie et les musiciens du Groupe des Six. 


\section{Raffinement et sophistication : une impasse?}

La mélodie est alors le genre artistique largement mis à l'honneur permettant de concilier poésie et musique. Les poètes du XIX ${ }^{e}$ siècle, Musset, Lamartine, Victor Hugo et Théophile Gautier sont abondamment mis en musique mais les poètes parnassiens et symbolistes Baudelaire, Leconte de Lisle, Verlaine, Henri de Régner - sont également très prisés en cette fin de siècle. C'est sans doute la poésie de Verlaine qui séduit le plus grand nombre de compositeurs du dernier tiers du XIX e siècle ; Fauré, Debussy et Reynaldo Hahn exploitent parfois les mêmes poèmes, notamment ceux empruntés au recueil des Fêtes galantes.

Le répertoire français s'enrichit de mélodies exploitant donc les textes les plus raffinés, riches de symboles, en métaphores subtiles, cherchant à échapper à la réalité du quotidien. La dimension sonore donnée par les compositeurs semble engendrer une parfaite fusion texte-musique. Reynaldo Hahn écrit lui-même : "Une seule chose m’intéresse, m’enthousiasme et m’obsède : la réunion de la littérature et de la musique $! »^{3}$ Proust qualifie Reynaldo Hahn de "poète chanteur et musicien " et évoque l'émotion qui le bouleverse en entendant chanter son ami compositeur-interprète :

Reynaldo Hahn [...] la tête légèrement renversée en arrière, la bouche mélancolique, un peu dédaigneuse, laissant s'échapper le flot rythmé de la voix la plus belle, la plus triste et la plus chaude qui ne fut jamais, cet "instrument de musique de génie" qui s'appelle Reynaldo Hahn étreint tous les cœurs, mouille tous les yeux, dans le frisson d'admiration qu'il propage au loin et qui nous fait trembler, nous courbe tous l'un après l'autre, dans une silencieuse et solennelle ondulation des blés sous le vent. ${ }^{4}$

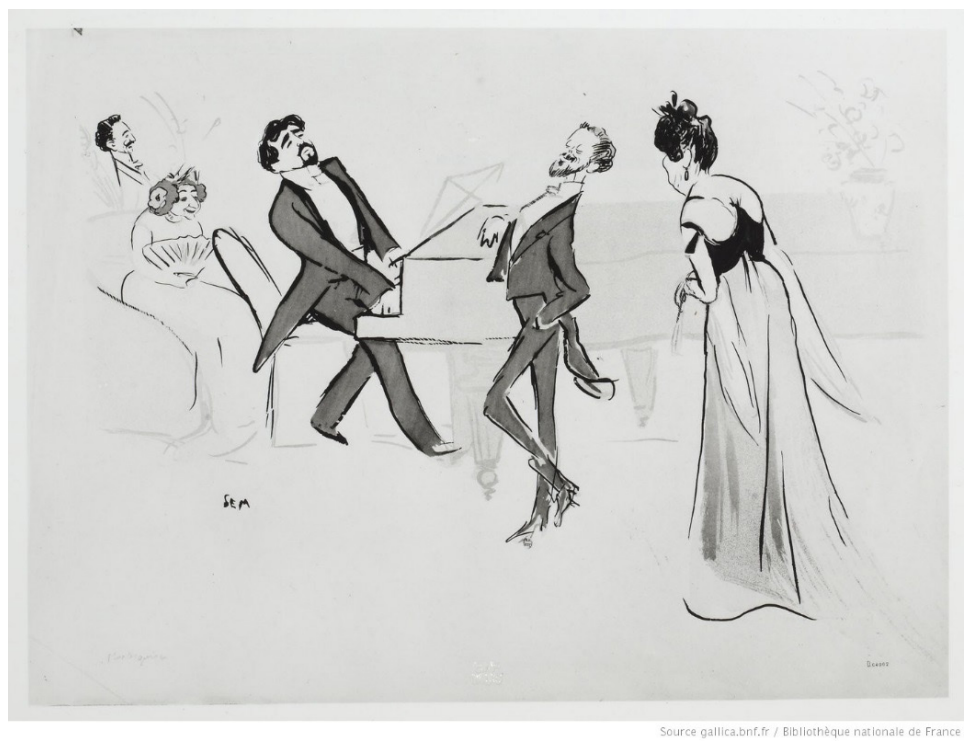

Figure 1. Sem : «Une soirée chez Madeleine Lemaire », caricature ; Reynaldo Hahn au piano. Photographie de la Bibliothèque nationale de France, Gallica 


\section{Nouvelles aspirations poétiques}

Les poètes du mouvement symboliste optent souvent pour le vers libre. Cette liberté de rythme séduit plusieurs compositeurs ; Debussy façonne à son tour lui-même les poèmes en vers libres de ses 4 mélodies Les proses lyriques (1892) ; l'adéquation entre musique et poésie paraît alors évidente et incontestable ; l'esthétique des poèmes manifeste une proximité étroite avec le mouvement symboliste, particulièrement proche de la veine de Verlaine, Maeterlinck, Mallarmé et Jules Laforgue.
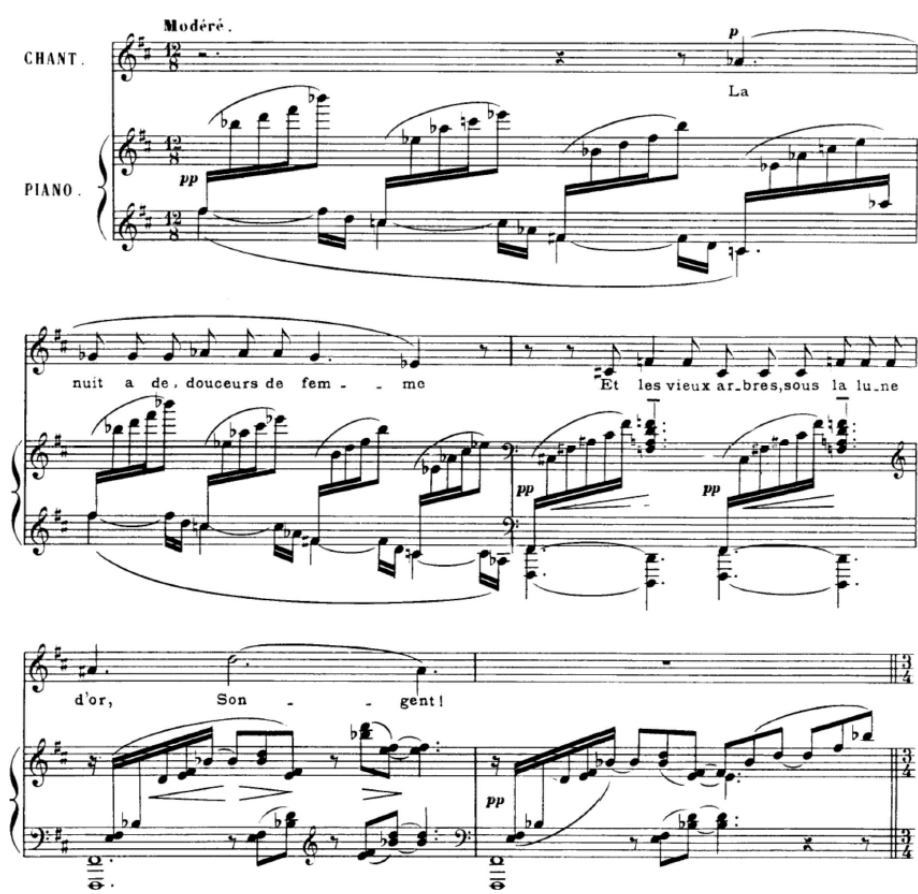

Figure 2. Debussy, Proses lyriques n ${ }^{\circ} 1$ " De rêve... ", mes. 1 à 5

À travers la sensualité diffuse et les harmonies capiteuses, la liberté du débit, la déclamation et les galbes mélodiques se marient merveilleusement au verbe ; cette symbiose parfaite unissant musique et poèmes reste probablement unique dans tout le répertoire de la mélodie puisqu'émanant du même artiste.

Vous auriez eu la joie d'entendre Melle Vallin chanter les Proses lyriques, et surtout Le Promenoir des amants. ${ }^{5}$. Il y avait de quoi pleurer, - comme dit à peu près votre ami Pelléas ! - je ne sais pas où elle va chercher cette prenante compréhension des courbes que décrit la musique à travers le texte... mais c'est absolument beau ! et très simple... ${ }^{6}$

"Les courbes que décrit la musique à travers le texte... " Quelle belle formule du musicien révélant la conception des rapports entre musique et poésie! 
Avec l'utilisation de la prose, c'est une nouvelle dimension des liens entre musique et poésie qui s'ouvre soudain. Pierre Louÿs s'interroge en 1890 dans son Journal intime:

Qui donc [...] saura rythmer un chant si juste, que le compositeur n'ait plus qu'à le transcrire et à l'orchestrer, comme en sous-ordre ; fera des poèmes si définitifs que la musique sur chacun des chants n'ait plus qu'une apparence d'accompagnement servile et discret, et que l'œuvre puisse dûment, et orgueilleusement s'intituler drame lyrique, avec musique de scène? (Louÿs 1973, 340)

Dans Les chansons de Bilitis (1897-98), Debussy franchit cette nouvelle étape vers l'originalité des rapports musique/poésie en mettant en musique la prose rythmée de son ami Pierre Louÿs - les poèmes étaient prétendument écrits par une poétesse antique et traduits du grec. Le recueil parait en 1894 ; à la demande de l'écrivain, Debussy illustre trois de ces poèmes en musique : "La flûte de Pan ", "La chevelure ", «Le tombeau des Naïades ".7 Ces mélodies voient le jour en 1897 pour les deux premières, en mars 1898 pour la troisième; le cycle parait en 1899. Le compositeur trouve dans ces poèmes la totale liberté du débit de la prose rythmée et parvient à modeler une déclamation vocale quasi recitativo, très proche de celle de Pelléas et Mélisande; la souplesse de l'arabesque mélodique prolonge alors parfaitement la ductilité de la prose de Louÿs. Le poème de la première de ces mélodies "La flûte de Pan " donne l'esprit général du cycle :

Pour le jour des Hyacinthies,

Il m’a donné une syrinx

Faite de roseaux bien taillés,

Unis avec la blanche cire

Qui est douce à mes lèvres comme le miel.

Il m’apprend à jouer, assise sur ses genoux ;

Mais je suis un peu tremblante.

Il en joue après moi,

Si doucement que je l'entends à peine.

Nous n'avons rien à nous dire,

tant nous sommes près l'un de l'autre ;

mais nos chansons veulent se répondre,

et tour à tour nos bouches

s’unissent sur la flûte.

Il est tard,

voici le chant des grenouilles vertes

qui commence avec la nuit. 


\section{Ma mère ne croira jamais \\ que je suis restée si longtemps \\ à chercher ma ceinture perdue.}

Ces poèmes en prose sont empreints d'une sensualité très suggestive où affleure l'érotisme. "Bilitis est licencieuse [...] avec une parfaite candeur » écrit Pierre Louÿs (Debussy 2005, 253) ; il ajoute à Debussy en 1895 " Je peux te faire de la prose très pleine et très rythmée qui vaudra largement les vers de M. Gallet. (Bilitis est écrite tout entière en vers de 8 syllabes non rimées, vérifie.)»(Debussy 2005, 248-249). Et Debussy répond :

J'aime mieux la prose rythmée que les vers (au moins pour la musique) ça rebondit davantage et on n'est plus arrêté par des tas de considérations. D'ailleurs, la musique et les vers, c'est deux chansons qui cherchent vainement à s'accorder, même dans les cas très rares où cela s'accorde, cela fait l'effet d'un mauvais calembour. ${ }^{8}$

L'extrême raffinement et la sensualité du langage harmonique de Debussy, teintées de modalité, de balancements harmoniques, de parallélismes et d'agrégats, offrent un prolongement voluptueux aux poèmes. Pierre Loÿs écrit à son ami compositeur : «Ce que tu as fait sur mes Bilitis est adorablement bien ; tu ne peux pas sentir le plaisir que j'en ai. »?

De la belle poésie romantique au vers libre puis à la prose, la musique vocale de Debussy marque donc une étape décisive dans l'évolution des rapports du texte à la musique.

\section{Le nationalisme}

Dès Août 1870, période des premiers affrontements militaires contre la Prusse, fleurit le genre de la chanson nationaliste puis bientôt revancharde - et cela jusqu'en 1918. Cette fibre nationaliste de la chanson irrigue alors toutes les couches de la société. Dans ce contexte historique et social nait tout un répertoire de chansons politiques et nationalistes teintées de gouaille, mais surtout d'amertume de la défaite, de haine de l'ennemi, de soif de revanche, dans la lignée des poèmes et chansons de Paul Déroulède et de chansonniers très populaires. Ce genre est alors principalement pratiqué dans les cafés, cafés concerts. De très nombreux paroliers et chansonniers s'illustrent dans ce genre très en vogue, mais les compositeurs de ' musique savante `, portés par le contexte de la première Guerre Mondiale, se laissent parfois tenter par la veine nationaliste et revancharde. Il suffira d'invoquer quelques exemples.

Ravel et Debussy illustrent cet aspect en façonnant à la fois texte et musique. C'est en demi-teinte et par allusions voilées que Ravel évoque les trois couleurs du drapeau français dans la seconde des 3 Chansons pour chour "Trois beaux oiseaux du paradis " (1915), chanson rythmée par les mots "Mon ami z’il est à la guerre".

Pendant la guerre, Debussy affirme de façon brutale que «Trente millions de Boches ne peuvent pas détruire la pensée française » et signait «musicien français »; quelques-unes de 
ses lettres montrent un nationalisme exacerbé et haineux... De façon beaucoup plus véhémente et volontairement déchirante, Debussy cherche à émouvoir le public dans « Le Noël des enfants qui n’ont plus de maison" (Décembre 1915).

Nous n'avons plus de maisons!

Les ennemis ont tout pris, tout pris, tout pris,

Jusqu'à notre petit lit!

Ils ont brûlé l'école et notre maitre aussi,

Ils ont brûlé l'église et monsieur Jésus-Christ,

Et le vieux pauvre qui n’a pas pu s'en aller!

Nous n'avons plus de maisons!

Les ennemis ont tout pris, tout pris, tout pris,

Jusqu’à notre petit lit!

Bien sûr ! Papa est à la guerre,

Pauvre maman est morte!

Avant d'avoir vu tout ça.

Qu'est-ce que l'on va faire?

Noël, petit Noël, n'allez pas chez eux, n'allez plus jamais chez eux, punissez-les !

Vengez les enfants de France!

Les petits Belges, les petits Serbes, et les petits Polonais aussi !

Si nous en oublions, pardonnez-nous.

Noël ! Noël ! surtout, pas de joujoux,

Tâchez de nous redonner le pain quotidien.

Nous n'avons plus de maisons!

Les ennemis ont tout pris, tout pris, tout pris.

Jusqu'à notre petit lit!

Ils ont brûlé l'école et notre maittre aussi,

Ils ont brûlé l'église et monsieur Jésus-Christ,

Et le vieux pauvre qui n'a pas pu s'en aller!

Noël! Écoutez-nous, nous n’avons plus de petits sabots !

Mais donnez la victoire aux enfants de France.

Debussy écrit à Paul Dukas :

Pour le côté devoir : j'ai organisé trois séances de musique au profit d'œuvres de guerre. Au point de vue financier, ça a très bien marché. J'ai pu constater aussi combien notre public reste attaché aux mêmes romances! Le succès de ces trois séances a été pour un Noël des enfants qui n'ont plus de maison - paroles et musique de C. Debussy... vous voyez ça d'ici : «La maman est morte; Papa est à la guerre; nous n'avons plus de petits sabots; nous aimons mieux du pain que des joujoux » et pour conclure : «la victoire 
aux enfants de France. Ça n'est pas plus malin que ça ? Seulement ça entre tout droit dans le cour des citadins $»{ }^{10}$

Puis il ajoute quelques temps après : "Je vous y envoie le Noël, pour lequel vous voudrez bien avoir de l'indulgence ! il a été écrit la veille du jour où je fus opéré. ${ }^{11}$

Cette mélodie, faussement naïve, patriotique et revancharde, est conçue pour émouvoir de façon immédiate ; elle traduit aussi les sentiments nationalistes et parfois haineux du compositeur à cette époque. Debussy précise du reste : "C'est ma seule manière à moi de faire la guerre... »(Busser 1955, 204)

\section{Entre salon et boulevard - La gouaille française}

Hanns Eisler (1898-1962), disciple de Schoenberg et penseur de la musique et de ses rapports avec la société déclare dans un essai :

L'une des caractéristiques de cette crise musicale est la séparation entre musique légère et musique sérieuse. Cette séparation n'est-elle pas très curieuse? Ne peut-on se divertir qu'avec la pire camelote musicale? Sommes-nous obligés d'arborer une mine sérieuse et des allures de snob quand nous écoutons de la musique classique sérieuse ? (Eisler $1998,120)$

Le contexte de la $\mathrm{III}^{\mathrm{e}}$ République suscite la naissance de tout un répertoire de chansons sociales, politiques et nationalistes, teintées d'humour, de gouaille et de goguenardise. Séduits par la chanson de cabaret et de boulevard, quelques compositeurs, cherchent à sortir du cadre étroit de la mélodie de salon et proposent une orientation nouvelle pour ce genre. La mélodie explore alors le domaine humoristique, ironique, léger et canaille, frôlant parfois le registre de la chanson grivoise ou gaillarde. Ces ouvres mettent en musique des textes sans prétention, parfois écrits par les compositeurs eux-mêmes.

Ces mélodies, ou doit-on dire ‘ chansons `, exploitent des poèmes ou des textes souvent empreints d'humour ou même franchement légers et cocasses. Emmanuel Chabrier (18421894), sans renoncer à la beauté pure des poèmes de Baudelaire, ${ }^{12}$ explore de nouvelles inspirations littéraires pour ses mélodies et clame cette préoccupation à son éditeur : "Moi, ma première préoccupation est de faire ce qu'il me plaît, en cherchant tout d'abord à dégager ma personnalité ; ma seconde est de ne point être emmerdant. " ${ }^{13}$

Pour la série de six mélodies ‘ animalières `, Chabrier sollicite des poèmes d'Edmond Rostand et de sa femme Rosemonde Gérard.

Villanelle des petits canards

Ballade des gros dindons 
Pastorale des cochons roses

L'île heureuse

Les cigales

Toutes les fleurs

Le poème d'Edmond Rostand de "La ballade des gros dindons " (fin 1889 14 ) donne le ton de l'ensemble du cycle :

Les gros dindons, à travers champs,

D'un pas solennel et tranquille,

Par les matins, par les couchants,

Bêtement marchent à la file,

Devant la pastoure qui file,

En fredonnant de vieux fredons,

Vont en procession docile,

Les gros dindons.

Ils vous ont l'air de gros marchands

Remplis d'une morgue imbécile,

De baillis rogues et méchants

Vous regardant d'un œil hostile :

Leur rouge pendeloque oscille;

Ils semblent parmi les chardons,

Gravement tenir un concile,

Les gros dindons.

N'ayant jamais trouvé touchants

Les sons que le rossignol file,

Ils suivent, lourds et trébuchants,

L'un d'eux, digne comme un édile ;

Et lorsqu'au lointain campanile

L’angélus fait ses lents : din ! dons !

Ils regagnent leur domicile,

Les gros dindons !

Prudhommes gras, leurs seuls penchants

Sont vers le pratique et l'utile,

Pour eux, l'amour et ses doux chants

Sont un passe-temps trop futile ;

Bourgeois de la gent volatile, 
Arrondissant de noirs bedons,

Ils se fichent de toute idylle,

Les gros dindons!

Dans une lettre de 1889 à ses éditeurs, Chabrier précise la portée de ce recueil de mélodies :

Ce que je ne veux pas, par exemple, ce sont ces éternels parterres en trois couplets où l'on cueille bêtement gratte-culs et chrysanthèmes ; ce que je ne veux pas, ce sont ces glaires où l'on s'aime au temps des petites fleurs ou pendant avril et mai [...] Moi, je voudrais faire gai, mais du gai pour les deux sexes ; du robuste, du bon enfant, des fables, des contes, enfin autre chose que Fauré et Holmès, et tous les autres en accolade. $^{15}$

Ces ‘ volailleries > sont, du reste, destinées à des interprètes-chanteuses de café-concert et d'opérette et se présentent comme de véritables caricatures de la société bourgeoise du XIX siècle, dans l'esprit de Grandville ou de Daumier. L'esprit du café-concert prévaut plus encore dans deux duos comiques avec orchestre, "Cocodette et Cocorico " et " Monsieur et Madame Orchestre ». Ces deux duos furent composés en 1878 pour le couple Bruet-Rivière qui chantaient à l'Eldorado, célèbre café-concert du boulevard de Strasbourg. "L'Ouvreuse de l'Opéra-Comique et de l'Employé du Bon Marché ", autre duo-bouffe pour soprano et ténor, d'une authentique veine comique, fut créé en 1888 chez Henriette Fuchs (18631927), cantatrice et critique musical. Chabrier y concilie parfaitement charme mélodique, tyrolienne burlesque, élégance du langage et humour populaire.

Les textes futiles et humoristiques choisis par Satie puis Poulenc s'inscrivent dans la continuation de la veine de Chabrier. Evoquons, notamment les Trois mélodies de Satie (1916) à l'esprit dadaïste. Dans la deuxième mélodie de ce recueil "Dis-moi, Daphénéo " (poème de Mimi Godebska), c'est un jeu de mot résolument simpliste, naïf et même juvénile qui est l'argument du poème :

Dis-moi, Daphénéo, quel est donc cet arbre

Dont les fruits sont des oiseaux qui pleurent?

Cet arbre, Chrysaline, est un oisetier.

Ah! Je croyais que les noisetiers

Donnaient des noisettes, Daphénéo.

Oui, Chrysaline, les noisetiers donnent des noisettes,

Mais les oisetiers donnent des oiseaux qui pleurent.

Ah !...

Par une répétition de formule mélodique ingénue comme celle d'une comptine et s'achevant dans une déclamation recto tono sur l'indication "presque blanc ", la musique traduit cette naïveté feinte et expire sur le "Ah ! » de Chrysaline. 


\section{Le café-concert}

Dans cette période de la III ${ }^{\mathrm{e}}$ République, après la guerre de 1870 et l'épisode dramatique de la Commune de Paris, parallèlement aux salons de l'aristocratie ou haute bourgeoisie parisienne fleurissent de nombreux cafés-concerts où se succèdent tour de chant, sketch, lecture de poèmes et pantomimes. Malgré l'anti-germanisme croissant, les musiciens savants, tiraillés entre deux directions esthétiques, vénèrent Wagner (Chabrier est un des piliers du cénacle Le Petit Bayreuth) et mettent cependant Verlaine en musique ; mais l'humour et la dérision trouvent également largement leur place dans cette société contrastée. Depuis le Second Empire, pendant la III ${ }^{\mathrm{e}}$ République, et notamment au tournant du siècle, des cafés concerts jouissent d'un succès vertigineux et drainent un public nombreux cherchant des divertissements légers.

Les établissements se multiplient ; l'Eldorado, l'Alcazar d'Été, Aux Ambassadeurs ${ }^{16}$, Cabaret des Quat'z Arts comptent parmi les plus connus ; les spectateurs de couches sociales extrêmement variées s'y pressent. Quelques chansonniers jouissent d'une réputation immense ; parmi les plus illustres, citons Aristide Bruant, Mac-Nab, Vincent Hyspa, Paulus, Paulette Darty, Yvonne Guilbert, Lucien Boyer ou Paul Delmet... Le Chat Noir, tenu par Rodolphe Salis de 1881-82 jusqu'à sa mort 1897, est un café-concert de taille très réduite, où revue musicale, expression littéraire et artistique, théâtre d'ombres trouvent leur place. Parmi les poètes célèbres qui fréquentent Le Chat Noir l'on compte Verlaine, Jules Jouy, Jean Lorrain, Alphonse Allais, Jehan Rictus ; des compositeurs fréquentent aussi le cabaret. Chansonniers et poètes y déclament des textes ; les satires politiques alternent avec les chansons humoristiques accompagnées au piano, sans prétention poétique, sans sophistication aucune. Du texte de divertissement.

Quelques compositeurs célèbres, tels que Debussy et Satie ont fréquenté ces cafés-concerts. Les compositions écrites pour ces établissements de divertissement instaurent une sorte de trait d'union entre mélodie savante et chanson de boulevard mais traduisent aussi une préoccupation sociale : rapprocher l'art de la vie quotidienne et des couches populaires.

C'est en partie par nécessité matérielle que Satie a été contraint d'endosser l'habit d'artiste de cabaret une bonne partie de sa vie, de produire des mélodies frivoles, souvent sur des textes humoristiques, chansons légères ou scabreuses, de " rudes saloperies " selon ses dires... Le succès de ces chansons tient sans doute plus aux paroles tandis que les caractéristiques musicales se plient souvent aux topoï du genre que Satie exploite avec recul, dérision et distanciation : valses lentes, mélodies très simples et suaves, forme à refrain, syncopes empruntées au ragtime... Quoique plus tard il ait dénoncé toute cette production comme étant contre sa nature, ces mélodies ont été parfois le versant le plus populaire de sa production.

La collaboration avec l'acteur, auteur, humoriste, chanteur Vincent Hyspa a été abondante ; Hyspa écrit et chante les textes, Satie met en musique ; parmi les plus célèbres : «L'omnibus automobile » (1900), "Un dîner à l'Elysée » (1900), « Le veuf » (1900), «Tendrement » (1902), valse lente au succès immédiat, "Chez le docteur » (1905), paroles tour- 
nant en dérision les lois Combes, et le " Petit recueil des fêtes », chanté par Hyspa au Cabaret des Quat'z Arts.

Paulette Darty n'est qu'interprète mais son charme universellement reconnu et sa voix ont permis le très large succès de chansons dont Satie a écrit la musique. La « reine de la valse lente > interprète particulièrement "Je te veux » (1902 - paroles d'Henry Pacory), valse modérée d'une trivialité délibérée. P. Darty chantera également en 1904 "La diva de l'empire », intermezzo américain.

Dans cet esprit boulevardier, cocasse mais trivial, la chanson de Satie sur un texte de D. Durante «Allons-y Chochotte, Chochotte allons-y (1905) reste l’une des plus célèbre :

Lorsque je vis Chochotte,

Elle me plut carrément ;

J'lui dis : «Êtes-vous mascotte? »

"Mais monsieur certainement."

"Alors sans plus attendre

Je veux être votre époux. "

Elle répond d'un air tendre :

"Je veux bien être à vous,

Mais pour cela, il vous faudra

Demander ma main à papa. »

Allons-y Chochotte, Chochotte...

Allons-y Chochotte, Chochotte allons-y.

Le soir du mariage,

Une fois rentrés chez nous,

J'prends la fleur de son corsage

Je fourre mon nez partout.

"Alors» me dit ma femme,

«Avant tout, écoute-moi.

J'vais couronner ta flamme.

Puisque tu m'aimes, prends-moi.

Mais pour cela il te faudra

Ne pas m'chatouiller sous le bras. "

Allons-y Chochotte [...]

V'là qu'au moment d'bien faire,

On entend sur l'boul'vard

Un refrain populaire

Et comme un bruit de pétards.

C'est une sérénade

Que donnent en notre honneur 
Une bande de camarades

Qui travaillent tous en chœur,

S'accompagnant des instruments

En carton, à cordes, à vent.

Allons-y Chochotte [...]

Le lendemain, Chochotte

Me dit : " Mon p'tit Albert,

J'veux un fils qui dégote

Mozart et Meyerbeer.

Pour en faire un prix de Rome

$T$ 'achèteras un phono

Que tu r'monteras mon p'tit homme

Au moment psycholo...

Et l'on marchera de ce moment là

Comme ton cylindre l'indiquera. "

Allons-y Chochotte [...]

Neuf mois après, Chochotte

Me rend papa d'un garçon.

"Ah! " s'écrie «Saperlotte »

La sage-femme "Mais que vois-je donc? "

"Qu'avez-vous donc, Madame?

Pourquoi crier si haut?"

Lui d'manda ma femme.

"N'a-t-il pas tout ce qu'il faut?»

Oui, mais voilà, on peut lire là

Sur son p'tit nombril ce refrain-là :

Allons-y Chochotte [...]

Suivant un parcours révélateur de l'évolution des goûts littéraires, Reynaldo Hahn, musicien du raffinement mélodique qui a principalement évolué dans les salons aristocratiques de la Princesse de Polignac ou bourgeois de Madeleine Lemaire, après avoir principalement mis en musique Verlaine et Lecomte de Lisle, s'oriente à partir des années 20 vers l'opérette, puis collabore avec Sacha Guitry pour des comédies musicales, confiant l'un des rôles de $\hat{O}$ mon bel inconnu! ${ }^{17}$ (1933) à Arletty, à une époque où le public est attiré par la comédie musicale et la musique noire américaine. 


\section{L'émergence du blues et du jazz}

La < musique nègre > arrive en Europe principalement avec les armées américaines à partir de 1917. Par étonnement, puis par engouement, musiciens et auditeurs vont s'intéresser à ces nouvelles expressions musicales. Dans un article, Ravel souligne l'importance du jazz sur sa musique :

A l'étranger, nous prenons le jazz au sérieux. Il exerce une influence sur notre œuvre. Le «blues » de ma Sonate, par exemple est du jazz stylisé, plus français qu’américain de caractère, peut-être, mais cependant fortement influencé par votre musique dite "populaire ».

Personnellement, je trouve le jazz extrêmement intéressant : les rythmes, le traitement de mélodies, les mélodies elles-mêmes. (Ravel 1989, 327)

Et il ajoute dans une interview :

Personne ne peut rejeter les rythmes aujourd'hui. La musique récente est pleine d'influences venues du jazz. Le fox-trot et les blue notes de mon opéra L'Enfant et les sortilèges n'en sont pas les seuls exemples. (Ravel 1989, 362)

Mais dans un premier temps, l'influence du jazz sur la musique savante se traduit en Europe, davantage dans le domaine de la musique instrumentale.

\section{Poulenc voyou}

Pour sa musique vocale, Francis Poulenc recourt à une extrême diversité d'inspiration poétique. D’une veine différente de celle de Satie, évoquant Paris aux relents prosaïques et malsains, la mélodie "L'anguille " des Quatre poèmes de Guillaume Apollinaire ${ }^{18}$ - février-mars 1931 - témoigne de l'esprit populaire spécifiquement parisien, aux accents de valse musette, et de "Poulenc avec l'accent parigot " ${ }^{19}$ (Bernac 1978, 63-64), "L'anguille, qui sent l'hôtel borgne, est rythmé à petits pas de chaussons de feutre et devrait émouvoir. Chanter cette mélodie sans ironie, en y croyant. » (Bernac 1978, 63-64) Pierre Bernac, l'interprète et ami, ajoute :

Attention! dans ce genre de mélodies, où Poulenc prend volontairement un accent faubourien, il est essentiel, tout en donnant son caractère à la mélodie, de n'être jamais vulgaire. Nul n'a mieux senti que Poulenc la sombre poésie d'une certaine sordide atmosphère parisienne. (Bernac 1978, 64-65)

Dès ses jeunes années, pour la Rapsodie nègre ${ }^{20}$ - 1917, en pleine période de guerre, Poulenc adopte le domaine de l'humour absurde et iconoclaste, allant jusqu'à la mystifica- 
tion. Le compositeur laisse transparaître des accents d'humour absurde dans la manière de Bibi-la-Bibiste de son amie Raymonde Linossier et des influences de l'art nègre qui commençait à prospérer. L'œuvre fut créée et chantée par le compositeur ; l'intermède vocal de l'œuvre exploite un texte de Makoko Kangourou ${ }^{21}$, en langue africaine imaginaire ; le texte n'est qu'une supercherie littéraire rédigé dans un langage dit de ‘ petit nègre `, utilisant une syntaxe volontairement fautive mais écrit par Marcel Prouille :

\section{Honoloulou, poti lama! \\ Honoloulou, Honoloulou, \\ Kati moko, mosi bolou \\ Ratakou sira, polama}

Cet intermède apparaît donc comme une sorte de mystification au caractère ludique et absurde, mais aussi provocateur et insolente d'un jeune compositeur qui cherche à se faire connaître. La dédicace à Erik Satie en est hautement symbolique et révélatrice de la distanciation, de l'humour et de la dérision. Cette œuvre heurta du reste Paul Vidal, maître de l'ancienne école académique.

Les chansons gaillardes de Poulenc forment un cycle de huit mélodies composées entre 1922 et 1926 dans l'euphorie et l'après-guerre, sur des textes anonymes du XVII ${ }^{e}$ siècle. Deux textes (chansons 2 et 5), plus tardifs, proviennent d'une Anthologie françoise publiée en 1765. Claude Rostand écrivait dans un article qu' «il y a chez lui [Poulenc] deux aspects essentiels, différents, même assez opposés : il y a le musicien aimable, divertissant, souriant, impertinent voire inconvenant [...], et il y a le musicien sévère, grave, qui n’a pas honte de son émotion et atteint à la grandeur " (Rostand 1950). Dans Les chansons gaillardes (19221926), Francis Poulenc aborde les thèmes les plus scabreux, mais avec une égale crudité chic: "J'ai tenté de démontrer que l'obscénité peut s'accompagner de la belle musique " (Poulenc 1993, 16) affirme-t-il. Evoquant l'ensemble du cycle, Poulenc déclare encore :

J'y tiens peut-être plus qu'à n'importe quoi de ce que j'ai fait. Il me semble que c'est très uniquement "Poulenc" et que personne n’aurait pu réussir ce genre de chansons mi-érotiques mi-élégiaques. (Poulenc 1998, 273)

Huit chansons composent le cycle :

"La maîtresse volage "

"Chanson à boire"

"Madrigal»

"Invocation aux Parques»

"Couplets bachiques"

« L'offrande » 
"La belle jeunesse"

"Sérénade»

Pierre Bernac évoque la "franchise truculente [de Poulenc] dans ses Chansons gaillardes, dont les textes sont toujours très lestes, soit sur le double sens des mots, soit en appelant très crument les choses par leur nom " (Bernac 1978, 194-195). Le prétexte littéraire ancien et érudit permet à Poulenc d'illustrer les textes les plus rabelaisiens sans aucune vergogne ; ces Chansons gaillardes sont parfaitement dignes de chansons de cabaret et de chansonniers ou renouent avec l'esprit truculent de certaines chansons polyphoniques françaises du XVI ${ }^{e}$ siècle, celles de Janequin particulièrement.

Illustrant l'intérêt nouveau des compositeurs pour le music-hall et le cinéma, comme Reynaldo Hahn écrit pour Arletty, Poulenc compose à plusieurs reprises pour Yvonne Printemps ${ }^{22}$ dont il évoque "le mirage de sa voix d'or ${ }^{23}$. Nait alors un répertoire conciliant les caractéristiques de la musique savante, de la comédie musicale et du music-hall, de la musique de scène et de la musique de film; ce répertoire est dédié le plus souvent à des comédiennes également chanteuses dont le charme et la voix ont conquis poètes et musiciens.

\section{Conclusion}

Ces lignes ont permis d'évoquer la période de 60 années où les compositeurs ont abordé des répertoires contrastés et des veines littéraires extrêmement variées, chantant la poésie dans divers registres, du raffinement le plus absolu à la chanson la plus triviale sans discrimination et sans dénigrer l'un ou l'autre genre. Les mutations sociales et politiques qui ont bouleversé cette période ont permis le voisinage d'un art de la quintessence de la sophistication, art de salon, avec un art plus populaire de café-théâtre et de boulevard ; cette la perméabilité nouvelle entre deux mondes et le glissement d'un genre vers l'autre a largement favorisé un renouveau des liens entre musique et poésie. Pendant cette période comprise entre la guerre de 1870 et les années 30, le raffinement de la musique et la gouaille du boulevard n'ont pas paru antinomique ; cette ambivalence des compositeurs s'achève sans doute avec l'essor du music-hall, du cinéma et la crise de 1929.

\section{Notes}

1 Yves Rassendren est maitre de conférences en Musicologie à l'Université Grenoble-Alpes et membre du laboratoire Luhcie.

2 Lettre de Duparc à Francis Jammes, 1904 (Duparc 1944, 63).

3 Reynaldo Hahn, lettre du 18 septembre 1894 à E. Risler (Blay 1993, 43). 
4 Marcel Proust, "La cour aux lilas et l'atelier aux roses : le salon de Mme Madeleine Lemaire ", essai concernant la vie mondaine, un article publié sous le pseudonyme de Dominique dans Le Figaro du 11 mai 1903.

5 Le Promenoir des deux amants est un cycle de trois mélodies, composé par Debussy en 1904 et 1910 sur des poèmes de Tristan l'Hermite.

6 Lettre de Debussy à André Caplet du 23 juin 1913 (Debussy 2005, 1630).

7 Les $30^{\mathrm{e}}, 31^{\mathrm{e}}$ et $46^{\mathrm{e}}$ poèmes du recueil de Pierre Louÿs qui en compte 156.

8 Lettre de Debussy à Pierre Louÿs du 10 avril 1895 (Debussy 2005, 249).

9 Lettre de Pierre Louÿs à Debussy du 7 juillet 1897 (Debussy 2005, 358).

10 Lettre à Paul Dukas, début avril 1917 (Debussy 2005, 2092-2093).

11 Lettre à Paul Dukas, du 17 mai 1917 (Debussy 2005, 2110-2111).

12 Emmanuel Chabrier, "L'invitation au voyage " pour voix, basson et piano, 1870.

13 Lettre de Chabrier à son éditeur Georges Costallat du 17 juillet 1887 (Chabrier 1994, 411).

14 Première édition : Paris, Enoch \& Costallat, 1890.

15 Lettre du samedi 29 juin 1889 à Enoch \& Costallat, éditeurs de la musique de Chabrier (Chabrier 1994, 648).

16 Rendu célèbre par les deux pastels de Degas.

17 Comédie musicale en trois actes, livret de Sacha Guitry. Première représentation à Paris, aux Bouffes-Parisiens, le 5 octobre 1933.

18 Apollinaire, poèmes extraits du recueil posthume Il y a (Paris 1925).

19 Propos retranscrits par Pierre Bernac.

20 Rapsodie nègre, composée en 1917, créée à Paris, au Théâtre du Vieux-Colombier, lors d'un concert Jane Bathori, le 11 décembre 1917. L’œuvre comporte cinq parties : Prélude, Ronde Honoloulou, Pastorale, Final ; seul l'intermède central sollicite à la voix.

21 Les Poésies de Makoko Kangourou, Paris : signé par un auteur prétendument du Liberia mais écrit par Marcel Prouille, (pseudonyme de Marcel Ormoy) en collaboration avec Charles Moulié que l'Académie Goncourt devait plus tard révéler sous le nom de Thierry Sandre. L'édition de 1910 est illustrée d'un frontispice de Frantz Calot, sous le pseudonyme de Guy Tollac, représentant le portrait caricaturé du supposé auteur.

22 En 1935, "À sa guitare " sur un poème de Ronsard, la mélodie extraite de la musique de scène composée pour La Reine Margot, pièce d'Édouard Bourdet, puis "Les chemins de l'amour ", "valse chantée ", en 1933, mélodie insérée dans la pièce Léocadia de Jean Anouilh.

23 Lettre à Pierre Fresnay de février 1949 (Poulenc 1998, 657).

\section{Bibliographie}

Armengaud, Jean-Pierre : Erik Satie. Paris : Fayard, 2009.

Bernac, Pierre : Poulenc et se mélodies. Paris : Buchet Chastel, 1978.

Blay, Philippe : «Douze lettres de Reynaldo Hahn». In : Bulletin Marcel Proust 43 (1993), 37-57. 
Busser, Henri : De Pelléas aux Indes galantes... de la flûte au tambour. Paris : Librairie Arthème Fayard, 1955.

Chabrier, Emmanuel : Correspondance. Éds R. Delage et F. Durif. Paris : Klincksieck, 1994.

Debussy, Claude : Correspondance. Paris : Gallimard, 2005.

Debussy, Claude : Monsieur Croche et autres écrits. Paris : Gallimard, 1987.

Duparc, Henri : Une amitié mystique, lettres d'Henri Duparc à Francis Jammes et Charles Bourdeu. Paris : Guy Ferchaut/Mercure de France, 1944.

Eisler, Hanns : Musique et société. Éd. A. Betz. Trad. D. Meur. Paris : Éd. de la Maison des Sciences de l'Homme, 1998.

Lacombe, Hervé : Francis Poulenc. Paris : Fayard, 2013.

Louÿs, Pierre: Euvres complètes. Vol. 9 : Journal intime (1929-1931). Genève : Slatkine, 1973.

Poulenc, Francis : Journal de mes mélodies. Paris : R. Machart/Cicero, 1993.

Poulenc, Francis : Correspondance 1910-1963. Éd. M. Chimènes. Paris : Fayard, 1998.

Prouille, Marcel (sous le pseudonyme de Marcel Ormoy) : Les poésies de Makoko Kangourou. Paris : Dorbon aîné, 1910.

Proust, Marcel (sous le pseudonyme de Dominique) : «La cour aux lilas et l'atelier des roses : le salon de Mme Madeleine Lemaire ». In : Le Figaro (11 mai 1903), 3.

Ravel, Maurice : Lettres, écrits et entretiens. Paris : Flammarion, 1989.

Rostand, Claude : «Première audition du Concerto de Francis Poulenc ». In : Paris-Presse (26 juillet 1950). 